\title{
Produção científica sobre a COVID-19 em bases de dados da área da saúde
}

\author{
PELLIZZON, Rosely de Fátima ${ }^{1 *}$ \\ DEMETRIO, Rosângela ${ }^{1,2^{* *}}$ \\ MONTERO, Edna Frasson de Souza $1,2,3^{* * *}$ \\ ${ }^{1}$ Núcleo de Comunicação Científica em Ciências da Saúde (NCCCS) \\ ${ }^{2}$ Universidade Federal de São Paulo - Brasil \\ ${ }^{3}$ Universidade de São Paulo - Brasil \\ ORCID ID: https://orcid.org/0000-0001-9270-9057* \\ ORCID ID: https://orcid.org/0000-0002-2610-5543** \\ ORCID ID: https://orcid.org/0000-0003-1437-1219***
}

\begin{abstract}
Resumo
Este trabalho objetiva identificar a instituição mais citada na produção científica sobre a COVID19. O levantamento deste estudo foi realizado nas bases de dados da área da saúde, de maior relevância, destacando-se PubMed, Scopus e Web of Science. No PubMed, o sistema não oferece índices bibliométricos para analisar as citações. Analisando as três primeiras instituições que receberam o maior número de citações em cada base de dados, o resultado mostra que na base de dados Scopus, identificou em primeiro lugar, com o total de 5.397 citações o Hospital Wuhan (China); em segundo lugar, o Guangzhou Institute of Respiratory Health (China), com 3.476 citações, e em terceiro, o Huazhong University of Science and Technology (Wuhan), que obteve 1.219 citações. Na base Web of Science, destacou-se em primeiro lugar, a University of London, que foi citada 841 vezes. Em segundo lugar, com 793 citações, a University of California System, e em terceiro, a Chinese University of Hong Kong, citada 339 vezes.
\end{abstract}

Palavras-chave: COVID-19. Base de dados. Bibliometria. Pandemias. Produção científica

\section{Scientific production about covid-19 in health area databases}

\begin{abstract}
This work aims to identify the institution most cited in the scientific production on COVID-19. The search for this study was carried out in the most relevant health databases, with emphasis on PubMed, Scopus and Web of Science. In PubMed, the system does not offer bibliometric indexes for analyzing citations. When analyzing the first three institutions that received the highest number of citations in each database, the result shows that, in the Scopus database, Wuhan Hospital (China) was identified in first place, with a total of 5,397 visits; second, the Guangzhou Institute of Respiratory Health (China), with 3,476 citations, and third, the Huazhong University of Science and Technology (Wuhan), which obtained 1,219 citations. In the Web of Science database, the University of London stood out in first place, being cited 841 times. Second, with 793 citations, the University of California System, and, third, the Chinese University of Hong Kong, cited 339 times.
\end{abstract}

Keywords: COVID-19. Database. Bibliometrics. Pandemics. Scientific Production.

\section{Producción científica sobre covid-19 en bases de datos del área de salud}

\begin{abstract}
Resumen
Este trabajo tiene como objetivo identificar la institución más citada en la producción científica sobre COVID-19. La búsqueda de este estudio se realizó en las bases de datos del área de salud, de mayor relevancia, destacando PubMed, Scopus y Web of Science. En PubMed, el sistema no ofrece índices bibliométricos para analizar citas. $\mathrm{Al}$ analizar las tres primeras instituciones que recibieron el mayor número de citas en cada base de datos, el resultado muestra que en la base de datos Scopus, el Hospital de Wuhan (China) fue identificado en primer lugar, con un total de 5.397 citas; en segundo lugar, el Instituto de Salud Respiratoria de Guangzhou (China), con 3.476 citas, y en tercer lugar, la Universidad de Ciencia y Tecnología de Huazhong (Wuhan), que obtuvo 1.219 citas. En la base de datos de Web of Science, la Universidad de Londres se destacó en primer lugar, que fue citada 841 veces. En segundo lugar, con 793 citas, la University of California System y, en tercer lugar, la Universidad China de Hong Kong, citada 339 veces.
\end{abstract}

Palabras clave: COVID-19. Base de datos. Bibliometrías. Pandemias. Producción Científica. 


\section{Introdução}

Em época de pandemia sobre a COVID-19, muitos estudos têm sido publicados nas fontes de informação científica. A divulgação dos resultados das pesquisas é importante para levar o conhecimento das evidências científicas que possam contribuir para o combate à COVID-19. Podese obter os dados da produção acadêmica, através da consulta, gerada pelas ferramentas de análises dos índices bibliométricos, que são oferecidos pelas bases de dados em estudo. Estes índices permitem quantificar, classificar e identificar autores, instituições e países com visibilidade em suas publicações. Nesse sentido, a produção científica mais citada e a instituição geradora da pesquisa poderão ter impactos significativos nos próximos estudos (AKERMAN, 2016; MUGNAINI, 2003).

\section{Metodologia}

As bases pesquisadas para este estudo foram: PubMed (NLM), Scopus (Elsevier) e Web of Science (Clarivate Analytics). Os levantamentos nas bases de dados foram realizados para o período de janeiro a agosto de 2020, sem restrição de idioma, utilizando a estratégia de busca apenas na recuperação do campo de título do artigo publicado: (SARS-CoV-2 OR COVID-19 OR 2019-nCoV OR 2019nCoV OR covid 19 OR covid19 OR coronavirus-2019 OR coronavirus OR corona-virus OR betacoronavirus OR coronavirus infections OR novel coronavirus OR nCoV OR coronavirinae OR coronaviridae).

\section{Resultados e Discussão}

Sobre a produção científica e total de citações recebidas, de acordo com as bases de dados, de janeiro a agosto de 2020, na PubMed foram publicadas 45.545 referências, porém o sistema da base não possibilita analisar quais artigos foram mais citados dentre esses trabalhos. Na Scopus, o total de trabalhos publicados foi de 42.110 e houve 63.032 citações. Na principal coleção da Web of Science, foram publicadas 28.985 referências, com 18.012 citações. Na Tabela 1 é demonstrado o total da produção científica e das citações recebidas nas três bases de dados.

Tabela 1. Produção científica e total de citações recebidas de acordo com as bases de dados

\begin{tabular}{lcc}
\hline \multicolumn{1}{c}{ BASE DE DADOS } & $\begin{array}{c}\text { TOTAL DE TRABALHOS } \\
\text { (jan.-ago. 2020) }\end{array}$ & CITAÇÕES \\
\hline PubMed & 45.545 & 63.032 \\
\hline Scopus & 42.110 & 18.012 \\
\hline Web of Science & 28.985 & 2 \\
\hline \\
\hline
\end{tabular}


Fonte: Elaboração das autoras (2020).

Na Tabela 2 são demonstradas as produções científicas das Bases de dados Scopus e Web of Science, de acordo com as instituições, trabalhos, citações e seus respectivos países de origem.

Tabela 2. Produção científica distribuída entre as instituições e país de origem e de citações recebidas

\begin{tabular}{lcccc}
\hline \multirow{2}{*}{$\begin{array}{c}\text { BASE DE } \\
\text { DADOS }\end{array}$} & INSTITUIÇÕES & $\begin{array}{c}\text { TOTAL DE } \\
\text { TRABALHOS } \\
\text { (Jan.-Ago. 2020) }\end{array}$ & CITAÇÕES & PAÍS \\
\hline \multirow{3}{*}{ Scopus } & Harvard Medical School & 765 & 6.309 & Estados Unidos \\
\cline { 2 - 5 } & $\begin{array}{c}\text { Huazhong University of } \\
\text { Science and Technology }\end{array}$ & 757 & 28.789 & China \\
\cline { 2 - 5 } & Tongji Medical College & 726 & 27.934 & China \\
\hline \multirow{3}{*}{ Web of Science } & University of London & 862 & 7.112 & Inglaterra \\
\cline { 2 - 5 } & Harvard University & 789 & 5.483 & Estados Unidos \\
\cline { 2 - 5 } & $\begin{array}{c}\text { University of California } \\
\text { System }\end{array}$ & 621 & 5.417 & Estados Unidos \\
\hline
\end{tabular}

Fonte: Elaboração das autoras (2020).

A Tabela 3 mostra o total de trabalhos publicados na Scopus e na Web of Science, as instituições que ficaram nos três primeiros lugares em maior número de citações de cada base e seus respectivos países de origem (MENDES-DA-SILVA, 2020; RHEE, 2015; BAI, 2016).

Tabela 3. Relação das instituições mais citadas e seus países de origem

\begin{tabular}{ccccc}
\hline \multirow{2}{*}{$\begin{array}{c}\text { BASE DE } \\
\text { DADOS }\end{array}$} & $\begin{array}{c}\text { TOTAL DE } \\
\text { TRABALHOS } \\
(\text { Jan.-Ago. 2020) }\end{array}$ & INSTITUIÇÕES & CITAÇÕES & PAÍS \\
\hline \multirow{2}{*}{ Scopus } & 42.110 & $\begin{array}{c}\text { Hospital Wuhan } \\
\text { Respinatory Health }\end{array}$ & 3.397 & China \\
\cline { 3 - 5 } & \multirow{2}{*}{$\begin{array}{c}\text { Huazhong University of } \\
\text { Science and Technology }\end{array}$} & 1.219 & China \\
\hline \multirow{2}{*}{$\begin{array}{c}\text { Web of } \\
\text { Science }\end{array}$} & 28.664 & University of London & 841 & China \\
\cline { 3 - 5 } & & University of California System & 793 & Estados Unidos \\
\cline { 3 - 5 } & & Chinese University of Hong Kong & 339 & China \\
\hline
\end{tabular}

Fonte: Elaboração das autoras (2020). 
Estas diferenças se devem ao fato de as bases de dados apresentarem diferentes formas de indexação e total de documentos. Ambas as bases de dados são multidisciplinares, reunindo várias disciplinas nas buscas. A Scopus indexa aproximadamente 24.600 títulos de revistas e mais de 5.000 editoras acadêmicas. Na Web of Science, possui em torno de 22 mil revistas, além de conferências e livros, capítulos de livros, revisões, dentre outros documentos. Quanto à liderança da China, há que se levar em consideração que a COVID-19 começou nesse país, o que pode ter influenciado o resultado, tendo em vista que os artigos provenientes dessas instituições foram publicados logo no início da pandemia (BAI, 2016).

\section{Considerações finais}

$\mathrm{Na}$ base de dados Scopus, a instituição que mais recebeu citação foi o Hospital de Wuhan - China, onde se iniciou a pandemia. Entretanto, na base de dados da Web of Science, a instituição foi a University of London - Inglaterra.

\section{Referências}

AKERMAN, M. Impacto na leitura de artigos científicos: uma tentativa de diálogo com "meu leitor”. Ciência \& Saúde Coletiva, v. 21, n. 3, p. 947-954, 2016.

DOI: https://doi.org/10.1590/1413-81232015213.06372015.

BAI, X. et al. Identifying anomalous citations for objective evaluation of scholarly article impact. PLoS One, v. 11, n. 9, p. e0162364, 2016. Disponível em: https://journals.plos.org/plosone/article?id=10.1371/journal.pone.0162364. Acesso em: 15 maio 2020.

MENDES-DA-SILVA, W. O que faz um artigo ser mais citado? Revista de Administração Contemporânea, v. 24, n. 6, p. 507-513, 2020. DOI: https://doi.org/10.1590/19827849rac2020200167.

MUGNAINI, R. A bibliometria na exploração de bases de dados: a importância da linguística. Transinformação. v. 15, n. 1, p. 45-52, 2003. DOI: https://doi.org/10.1590/S0103$\underline{37862003000100003 .}$.

PUBMED. Bethesda (MD): National Library of Medicine (US). [2020]. Disponível em: https://pubmed.ncbi.nlm.nih.gov/ Acesso em: 27 jul. 2020.

RHEE, J. S. High-impact articles-citations, downloads, and altmetric score. JAMA Facial Plastic Surgery, v. 17, n. 5, p. 323-324, 2015. DOI: https://doi.org/10.1001/jamafacial.2015.0869.

ROSSI, M. J.; BRAND, J. C. Journal article titles impact their citation rates. Arthroscopy, v. 36, n. 7, p. 2025-2029, 2020. DOI: https://doi.org/10.1016/j.arthro.2020.02.018.

SCOPUS. Netherlands: Elsevier. c2020. Disponível em: https://www-scopuscom.ez69.periodicos.capes.gov.br/search/form.uri?display=basic Acesso em: 22 jun. 2020. 
WEB OF SCIENCE. Boston (MA): Clarivate Analytics. c2020. Disponível em: http://appswebofknowledge.ez69.periodicos.capes.gov.br/WOS_GeneralSearch_input.do?product=WOS\&se arch $\_$mode $=$GeneralSearch $\&$ SID $=5$ D7gYRECNKleiIuWOFF\&preferencesSaved $=$ Acesso em: 22 jun. 2020.

\section{${ }^{1}$ Rosely de Fátima Pellizzon (São Paulo, São Paulo, Brasil)}

Mestra em Ciências pela Universidade Federal de São Paulo. Graduação em Biblioteconomia e Documentação. Bibliotecária especializada na Área da Saúde. Professora de Fontes de Informação Científica.

Contribuição de autoria Administração do projeto. Escrita - primeira redação. Investigação, Metodologia.

Lattes: http://lattes.cnpq.br/2647779315024108

E-mail: rosepellizzom@gmail.com

${ }^{2}$ Rosângela Demetrio (São Paulo, São Paulo, Brasil)
Mestre Profissional em Tecnologia, Gestão e Saúde Ocular pela Escola Paulista de Medicina / Unifesp. Graduações
em Comunicação Social e Jornalismo pela Universidade Anhembi Morumbi e Estudos Avançados da Língua
Inglesa. Atualmente atua como técnico administrativo em educação na Universidade Federal de São Paulo
Contribuição de autoria: Escrita - revisão, Visualização e Análise formal.
Lattes: $\underline{\text { http://lattes.cnpq.br/5887577007455394 }}$
E-mail: $\underline{\text { rodemetrio@gmail.com }}$

\section{${ }^{3}$ Edna Frasson de Souza Montero (São Paulo, São Paulo, Brasil)}

Graduação em Medicina pela Universidade Federal do Espírito Santo, doutorado em Técnicas Operatórias e Cirurgia Experimental pela Unifesp-EPM e Livre-docência. Docente e pesquisadora da FMUSP e UNIFESP. Atua no corpo editorial de várias revistas científicas.

Contribuição de autoria: Escrita - revisão, Visualização e Análise formal e Validação final.

Lattes: http://lattes.cnpq.br/2841222962564489

E-mail: edna.montero@gmail.com

\section{Avaliador(a) responsável:}

Eli Lopes da Silva

\section{Como citar este artigo:}

PELLIZZON, Rosely de Fátima, DEMETRIO, Rosângela, MONTERO, Edna Frasson de Souza. Produção científica sobre a COVID-19 em bases de dados da área da saúde. In: ABEC Meeting Live, 2020. Anais... São Paulo: Associação Brasileira de Editores Científicos, 2020. DOI: http://dx.doi.org/10.21452/abecmeeting2020.11 